\title{
PROPER DUBREIL-JACOTIN INVERSE SEMIGROUPS
}

\author{
by R. McFADDEN
}

(Received 12 March, 1974)

Introduction. This paper is concerned mainly with the structure of inverse semigroups which have a partial ordering defined on them in addition to their natural partial ordering. However, we include some results on partially ordered semigroups which are of interest in themselves. Some recent information $[1,2,6,7,11]$ has been obtained about the algebraic structure of partially ordered semigroups, and we add here to the list by showing in Section 1 that every regular integrally closed semigroup is an inverse semigroup. In fact it is a proper inverse semigroup [10], that is, one in which the idempotents form a complete class modulo the minimum group congruence, and the structure of these semigroups is explicitly known [5].

We then consider the structure of a proper inverse semigroup $S$ which is partially ordered in such a way that it is integrally closed and the canonical mapping $\mathscr{R}^{\natural}: S \rightarrow S / \mathscr{R}$ is isotone. Every such partial ordering is specified uniquely in terms of a partial ordering on the maximum group homomorphic image of $S$.

Finally, we turn to $F$-inverse semigroups, and, still under the assumption that $\mathscr{R}^{\natural}$ is isotone, prove that these are precisely those integrally closed inverse semigroups which are strongly integrally closed.

1. Regular Dubreil-Jacotin semigroups. A partially ordered semigroup is a semigroup $S$ with a partial ordering $\leqq$ such that

$$
x \leqq y, z \leqq w \text { imply that } x z \leqq y w \text { for all } x, y, z, w \in S .
$$

We shall use the notation and terminology of [3, Chapter 3$]$ for our discussion of partially ordered semigroups, and refer the reader to [3] for further details.

Let $S$ be a partially ordered semigroup. For each $x, y \in S$ the quasiresiduals of $x$ by $y$ are defined to be the sets

$$
\left\langle x \cdot{ }^{\prime} y\right\rangle=\{z \in S: y z \leqq x\},\left\langle x^{\cdot} \cdot y\right\rangle=\{z \in S: z y \leqq x\} .
$$

If $x \in S$ is such that $\langle x \cdot y\rangle=\left\langle x^{\cdot} \cdot y\right\rangle \neq \square$ for each $y \in S$, we write $\langle x: y\rangle$ for their common value and say that $x$ is equiquasiresidual.

We say that $x \in S$ is residuated if $\langle x . \cdot y\rangle$ and $\left\langle x^{\cdot}, y\right\rangle$ each contains a maximum element for each $y \in S$, and denote these maximum elements, the residuals of $x$ by $y$, by $x$.' $y$ and $x^{\cdot} \cdot y$ respectively. If $x, y=x \cdot y$ for each $y \in S$, we write $x: y$ for their common value and say that $x$ is equiresidual.

Two types of epimorphisms will concern us. In this section and in Section 2 we shall deal with a principal epimorphism of $S$ onto a partially ordered group $G$, by which we mean that $f$ is isotone and is such that the pre-image under $f$ of the negative cone of $G$ is a principal order ideal of $S$, that is, is of the form $[\leftarrow, \xi]=\{x \in S: x \leqq \xi\}$ for some $\xi \in S$. The following will then be taken as the definition of the term Dubreil-Jacotin semigroup [3, Theorem 25.3]. A partially ordered semigroup $S$ is a Dubreil-Jacotin semigroup if and only if it admits a principal 
epimorphic image which is a group. Such a group is necessarily unique up to isomorphism. If $S$ is a Dubreil-Jacotin semigroup then its core

$$
) S\left(=\bigcup_{x \in S}\langle x \cdot \cdot x\rangle \cup \bigcup_{x \in S}\left\langle x^{\cdot} \cdot x\right\rangle\right.
$$

has a maximum element $\xi$ which is equiquasiresidual, and the unique principal epimorphic group image of $S$ is given by $S / \mathscr{A}_{\xi}$, where $\mathscr{A}_{\xi}$ is the equivalence relation

$$
x \equiv y\left(\mathscr{A}_{\xi}\right) \Leftrightarrow\langle\xi: x\rangle=\langle\xi: y\rangle .
$$

The partial ordering $\mathrm{S} / \mathscr{A}_{\xi}$ is given by

$$
x \mathscr{A}_{\xi} \leqq y \mathscr{A}_{\xi} \Leftrightarrow\langle\xi: y\rangle \subseteq\langle\xi: x\rangle .
$$

We note that $\xi$ is the maximum element of $S$ mapped to the identity element of $G$.

In Section 3 we deal with a residuated epimorphism $f$ of $S$ onto a partially ordered group $G$, by which we mean that $f$ is isotone and the pre-image under $f$ of each principal order ideal of $G$ is a principal order ideal of $S$. The relevant facts for ou r pu rposes are the following.

$[3$, Theorem 25.4] Let $S$ be a partially ordered semigroup and let $f: S \rightarrow G$ be a principal epimorphism of $S$ onto a partially ordered group $G$. The following are equivalent :

(i) $f$ is residuated.

(ii) the maximum element $\xi$ of $) S$ (is residuated.

We say that a Dubreil-Jacotin semigroup $S$ is strong if the maximum element $\xi$ of ) $S$ (is residuated. In this case the unique residuated epimorphic group image of $S$ is given by $S / \mathscr{A}_{\xi}$, where $\mathscr{A}_{\xi}$ is the Molinaro equivalence relation

$$
x \equiv y\left(\mathscr{A}_{\xi}\right) \Leftrightarrow \xi: x=\xi: y .
$$

The partial ordering on $S / \mathscr{A}_{\xi}$ is given by

$$
x \mathscr{A}_{\xi} \leqq y \mathscr{A}_{\xi} \Leftrightarrow \xi: y \leqq \xi: x .
$$

Each class modulo $\mathscr{A}_{\xi}$ has a maximum element, the maximum element in the class of $x$ being $\xi:(\xi: x)$, and $x \mathscr{A}_{\xi} \leqq y \mathscr{A}_{\xi}$ if and only if $\xi:(\xi: x) \leqq \xi:(\xi: y)$.

[3, Theorem 25.6]. A partially ordered semigroup $S$ is a strong Dubreil-Jacotin semigroup if and only if there exists $\xi \in S$ such that $\xi$ is residuated and $[\leftarrow, \xi]=) S($.

In discussing inverse semigroups we shall use the terminology of [4], and make one deviation from the notation used there. It simplifies the notation here to denote the natural partial ordering [4, Vol. 2] on an inverse semigroup $S$ by $\preccurlyeq$ since most of our attention will be devoted to an additional partial ordering $\leqq$ on $S$. The definition of $\preccurlyeq$ may be given in the form $x \preccurlyeq y$ if and only if there exists $e=e^{2} \in S$ such that $x=e y$. Then $S(\preccurlyeq)$ is a partially ordered semigroup in which $x \preccurlyeq y$ if and only if $x^{-1} \preccurlyeq y^{-1}$, and $\preccurlyeq$ coincides, when restricted to the semilattice $E$ of idempotents of $S$, with the natural partial ordering on $E$. 
Every inverse semigroup has a minimum group congruence $\sigma$, defined by

$$
x \equiv y(\sigma) \Leftrightarrow \exists e \in E \quad \text { such that } e x=e y .
$$

If $E$ forms a complete $\sigma$-class then $S$ is said to be a proper inverse semigroup [10].

In fact, for a proper inverse semigroup $S$, we have $E=\left\{x \in S: x^{2} \preccurlyeq x\right\}$, and if $S$ has an identity element 1 then 1 is equiquasiresidual and $S(\preccurlyeq)$ is a Dubreil-Jacotin semigroup in which $\mathscr{A}_{1}=\sigma$.

We now proceed to study Dubreil-Jacotin semigroups $S$ which are regular, in the sense that for each $x \in S$ there exists $x^{\prime} \in S$ such that $x=x x^{\prime} x$ [4, Vol. 1].

PROPOSITION 1.1. Let $S$ be a regular Dubreil-Jacotin semigroup whose core has maximum element $\xi$. Then

(i) $x \leqq x \xi$ and $x \leqq \xi x$ for each $x \in S$.

(ii) $\xi^{2}=\xi$.

Proof. (i) Given $x \in S$, let $x^{\prime} \in S$ be such that $x=x x^{\prime} x$. Then $x x^{\prime}$ and $x^{\prime} x$ are idempotents, and so are congruent modulo $\mathscr{A}_{\xi}$ to $\xi$; therefore

$$
x=x\left(x^{\prime} x\right) \leqq x \xi \text { and } x=\left(x x^{\prime}\right) x \leqq \xi x .
$$

(ii) By (i), $\xi \leqq \xi^{2}$. Since $\xi^{2} \leqq \xi$ by definition of $\xi$, equality follows.

Although it implies that $\xi$ is the maximum idempotent of $S$, Proposition 1.1 does not provide a great deal of information about the structure of $S$, as Example 1 shows.

EXAMPLE 1. Let $X$ be an arbitrary partially ordered set with maximum element $\xi$, and consider the left zero semigroup $S$ on $X[4, \mathrm{Vol} .1]$. Certainly $S$ is a partially ordered semigroup, and for any $x, y \in S$,

$$
x^{\cdot} \cdot y=x, x \cdot y=\xi \text { if } y \leqq x,\langle x \cdot y\rangle=\square \quad \text { if } y \leq x .
$$

Since $S$ is regular, it follows that it is a regular (strong) Dubreil-Jacotin semigroup.

The situation is much better when $\xi$ is the identity element of $S$; Proposition 1.2 establishes a necessary and sufficient condition for this.

PROPOSITION 1.2. Let $S$ be a regular Dubreil-Jacotin semigroup whose core has maximum element $\xi$ : Then $\xi$ is the identity element of $S$ if and only if

$$
\check{\zeta}=x \cdot{ }^{\prime} x=x^{\cdot} \cdot x \text { for each } x \in S .
$$

Proof. For each $x \in S, x \xi \leqq x$ if and only if $\xi \in\langle x . \cdot x\rangle$. By definition of $\xi$, this is equivalent to $\xi$ being the maximum element of $\langle x \cdot \cdot x\rangle$, that is $\xi=x \cdot{ }^{\cdot} x$. It follows from Proposition 1.1 (i) that $\xi$ is a right identity element for $S$ if and only if $\xi=x$. $x$ for each 
$x \in S$. Similarly $\xi$ is a left identity element for $S$ if and only if $\xi=x^{\cdot} . x$ for each $x \in S$, and the result follows.

For the rest of this paper we shall consider only Dubreil-Jacotin semigroups $S$ for which $\xi$ is the identity element of $S$. We shall denote $\xi$ by 1 and $\mathscr{A}_{\xi}$ by $\mathscr{A}$, and call $S$ an integrally closed Dubreil-Jacotin semigroup. This is different from the nomenclature of [1], where it is assumed further that 1:x exists for each $x \in S$. In view of Theorem 1.6, the definition given here seems more natural. It is worthwhile pointing out that the term 'integrally closed' is usually applied only to residuated semigroups [3], but that we are not assuming the existence of all residuals, not even those of the form $1: x$.

LEMMA 1.3. Let $x \mathscr{A} \leqq y \mathscr{A}$ in $S / \mathscr{A}$, and let $y^{\prime} \in S$ be such that $y=y y^{\prime} y$. Then $y y^{\prime} x \leqq y$ and $x y^{\prime} y \leqq y$ in $S$; also $y y^{\prime} x \equiv x y^{\prime} y \equiv x(A)$.

Proof. By definition, $x \mathscr{A} \leqq y \mathscr{A}$ in $S / \mathscr{A}$ if and only if $y z \leqq 1$ implies that $x z \leqq 1$ and $z y \leqq 1$ implies that $z x \leqq 1$ in $S$. But $y^{\prime} y \leqq 1$; so $y^{\prime} x \leqq 1$ and $y y^{\prime} x \leqq y$. Similarly $x y^{\prime} y \leqq y$.

Lemma 1.4. On $E$, $\leqq$ coincides with $\preccurlyeq$.

Proof. If $e \leqq f$ in $E$, then

$$
e=e^{2} \leqq e f \leqq e 1=e ;
$$

so $e=e f$. Similarly $e=f e$; whence $e \preccurlyeq f$. Conversely, $e \preccurlyeq f$ implies that $e=e f \leqq 1 f=f$.

Lemma 1.5. If $E_{1}=\{x \in S: x \equiv 1(\mathscr{A})\}$ then $E_{1}=E$.

Proof. Since every idempotent of $S$ is congruent modulo $\mathscr{A}$ to 1 , we have $E \subseteq E_{1}$.

Suppose $x \equiv 1(\mathscr{A})$ and let $x^{\prime} \in S$ be such that $x=x x^{\prime} x$. Then $x^{\prime} \equiv 1(\mathscr{A})$ and since 1 is maximum in its $\mathscr{A}$-class, $x \leqq 1, x^{\prime} \leqq 1$. Therefore $x=x x^{\prime} x \leqq x 1 x=x^{2} \leqq x$, whence $x=x^{2}$ and $E_{1} \subseteq E$.

THEOREM 1.6. If $S$ is a regular integrally closed Dubreil-Jacotin semigroup, then $S$ is a proper inverse semigroup with identity element.

Proof. We prove that the idempotents of $S$ commute. As the identity class modulo $\mathscr{A}$, the set $E_{1}$ defined in Lemma 1.5 is a subsemigroup of $S$, and under the restriction to $E_{1}$ of the partial ordering on $S, E_{1}$ is a partially ordered semigroup with maximum element 1 . By Lemma 1.5, the same is true of $E$.

Now let $e, f \in E$. Then $e f \leqq e 1=e, e f \leqq 1 f=f$, while if $x \in E$ and $x \leqq e, x \leqq f$, then $x^{2}=x \leqq e f$. Therefore $e f=e \wedge f$, the greatest lower bound in $E$ of $e$ and $f$. Similarly $f e=e \wedge f$, and so $e f=f e$, that is $S$ is an inverse semigroup with identity element.

Now let $x, y \in S$ with $x \equiv y(\mathscr{A})$. Then $x^{-1} x, x x^{-1} \in E_{1}$ and $x^{-1} x \equiv x^{-1} y(\mathscr{A})$; whence $x^{-1} y \in E_{1}$. It follows that $x^{-1} y=g \in E_{1}, x x^{-1} y=x g$, and that there exist $f, g \in E_{1}$ such that $f y=x g$. But by Lemma $1.5, E_{1}=E$ in the inverse semigroup $S$, and therefore $x E=E x$ 
for each $x \in S$. We conclude that if $x \equiv y(\mathscr{A})$, then there exists $e \in E$ such that $e x=e y$, and so $\mathscr{A} \subseteq \sigma$. Since $\sigma$ is the minimum group congruence on $S$, we have $\mathscr{A}=\sigma$. Therefore the identity class modulo $\sigma$ is $E_{1}=E$, that is it consists only of idempotents, and so $S$ is a proper inverse semigroup and the proof is complete.

Notice that it follows from the proof of this theorem that a band with identity element 1 is a semilattice if and only if it can be made into a partially ordered semigroup with a maximum element 1.

COROLLARY. The partial orderings $\leqq$ and $\preccurlyeq$ coincide on $\sigma$-classes.

Proof. Since $x \preccurlyeq y$ implies that $x \leqq y$, we need only show that $x \preccurlyeq y$ whenever $x \leqq y$ and $x \equiv y(\sigma)$. But $x \equiv y(\sigma)$ implies that $x^{-1} x \equiv x^{-1} y(\sigma)$, and so $x^{-1} y \in E$, by Theorem 1.6. Therefore

$$
\begin{aligned}
x \leqq y & \Rightarrow x^{-1} x \leqq x^{-1} y \Rightarrow x^{-1} x \preccurlyeq x^{-1} y, \quad \text { by Lemma } 1.5 \\
& \Rightarrow x=x x^{-1} x \preccurlyeq x x^{-1} y \preccurlyeq y .
\end{aligned}
$$

Although $\sigma$ and $\mathscr{A}$ coincide on $S$, so that the groups $S / \sigma$ and $S / \mathscr{A}$ are algebraically isomorphic, in general they are not isomorphic as partially ordered groups. The partial ordering induced on $S / \sigma$ by $\preccurlyeq$ is always trivial, but the same may not be true of $S / \mathscr{A}$ and $\leqq$.

EXAMPLE 2. Let $\mathbb{Z}$ denote the additive group of the integers (both here and in Examples 3 and 4). Let $S=\mathbb{Z}$, and let $\leqq$ be the usual ordering on $\mathbb{Z}$. Then $S$ is a regular (strong) Dubreil-Jacotin semigroup on which both $\sigma$ and $\mathscr{A}$ are equality. But $S / \sigma \simeq \mathbb{Z}$ with the trivial partial ordering, while $S / \mathscr{A} \simeq \mathbb{Z}$ with the usual ordering. Although $\preccurlyeq$ and $\leqq$ coincide on each $\sigma$-class, and $x \preccurlyeq y$ implies that $x \leqq y$, the two partial orderings are distinct.

2. Integrally closed Dubreil-Jacotin inverse semigroups. We now turn to the problem of classifying the partial orderings on regular integrally closed Dubreil-Jacotin semigroups. Since each of these semigroups is, by Theorem 1.6, an inverse semigroup, from now on we shall call them integrally closed Dubreil-Jacotin inverse semigroups [1]. The problem, then, is that of determining the partial orderings on a proper inverse semigroup $S$ under which $S$ is an integrally closed Dubreil-Jacotin inverse semigroup. We have not been able to do this in general, but only, following Blyth [1], for partial orderings $\leqq$ under which Green's relation $[4$, Vol. 1$] \mathscr{R}$ is regular $[3$, Chapter 1$]$ in the sense that

$$
x \leqq y \Rightarrow x x^{-1} \leqq y y^{-1} .
$$

This condition is satisfied for $\preccurlyeq$ in any inverse semigroup, as is the corresponding condition for $\mathscr{L}$. In general it is not satisfied for $\leqq$, and we shall see in Example 4 that the conditions for $\mathscr{R}$ and $\mathscr{L}$ are independent of each other.

In any inverse semigroup $S$ we have $x \equiv y(\mathscr{R})$ if any only if $x x^{-1}=y y^{-1}$; the relation defined on $S / \mathscr{R}$ by $x \mathscr{R} \leqq y \mathscr{R}$ if and only if $x x^{-1} \leqq y y^{-1}$ is a partial ordering on $S / \mathscr{R}$, and to say that $\mathscr{R}$ is regular is to say that the canonical mapping $\mathscr{R}^{\natural}: S \rightarrow S / \mathscr{R}$ is isotone. 
Assuming that $\mathscr{R}$ is regular under $\leqq$, we obtain Theorem 2.1. This is a generalization of Blyth's Theorem 8 [1]; for in the first place we assume only that $\mathscr{R}$ is regular under $\leqq$ and so obtain non-trivial partial orderings on the group in question. In the second, the semigroups considered in [1] are in fact $F$-inverse semigroups [8] (by definition, an inverse semigroup is an $F$-inverse semigroup if and only if each $\sigma$-class contains a maximum element under $\preccurlyeq$ ) and although each $F$-inverse semigroup is necessarily proper and has an identity element, the class of proper inverse semigroups with identity element is much wider than that of $F$-inverse semigroups, even with the additional partial ordering.

Proper inverse semigroups are exactly those in which $\sigma \cap \mathscr{R}$ is equality. Using this, D. B. McAlister [5] has determined the structure of all proper inverse semigroups, as follows.

If $X$ is a partially ordered set and $a, b \in X$, then the notation $a \wedge b \in X$ means that $a$ and $b$ have a greatest lower bound $a \wedge b$ in $X$. If $Y \subseteq X$ and $a, b \in Y$, the notation $a \wedge b \in Y$ means that $a$ and $b$ have a greatest lower bound $a \wedge b$ which is an element of $Y$. A non-empty subset $Y$ of $X$ is a subsemilattice of $X$ if the condition $a, b \in Y$ implies that $a \wedge b \in Y$, and $Y$ is an ideal of $X$ if $a \in X, \mathrm{~b} \in Y$ and $a \leqq b$ imply that $a \in Y$.

Let $X$ be a partially ordered set, let $Y$ be a subsemilattice of $X$ which is also an ideal of $X$, and let $G$ be a group which acts (on the left) on $X$, by order automorphisms. Suppose that $G . Y=X$ and that $g Y \cap Y \neq \square$ for each $g \in G$. Then the set

$$
P(G, X, Y)=\left\{(a, g) \in Y \times G: g^{-1} a \in Y\right\}
$$

with the product

$$
(a, g)(b, h)=(a \wedge g b, g h)
$$

is an inverse semigroup.

[5, Proposition 1.2]. In $P=P(G, X, Y)$ as above,

(i) $(a, g)^{-1}=\left(g^{-1} a, g^{-1}\right)$;

(ii) the idempotents are the elements $(a, 1), a \in Y$ : they form a semilattice isomorphic to $Y$;

(iii) $(a, g) \mathscr{R}(b, h) \Leftrightarrow a=b,(a, g) \mathscr{L}(b, h) \Leftrightarrow g^{-1} a=h^{-1} b$;

(iv) $(a, g) \sigma(b, h) \Leftrightarrow g=h$; thus $G \simeq P / \sigma$;

(v) $(a, g) \preccurlyeq(b, h) \Leftrightarrow g=h$ and $a \leqq b$ in $Y$;

(vi) $P$ has an identity element if and only if $Y$ has a maximum element;

(vii) the $\sigma$-class of $(a, g)$ contains a maximum element if and only if $1 \wedge g 1$ exists; the maximum element in that case is $(1 \wedge g 1, g)$.

[5, Theorem 2.6]. Let $X$ be a partially ordered set and let $Y$ be an ideal and subsemilattice of $X$. If $G$ is a group which acts on $X$ by order automorphisms, then $P(G, X, Y)$ is a proper inverse semigroup.

Conversely, any proper inverse semigroup is isomorphic to $P(G, X, Y)$ for some partially ordered set $X$ with ideal and subsemilattice $Y$ and some group $G$ which acts on $X$ by order automorphisms.

We may now assume that every integrally closed Dubreil-Jacotin inverse semigroup is of the form $P(G, X, Y)$ as described above, and that $Y$ has a maximum element 1 . 
THEOREM 2.1. Let $P=P(G, X, Y)$ be a proper inverse semigroup with identity element. There is a one-to-one correspondence between partial orderings on $P$ for which $\mathscr{R}$ is regular and $P$ is an integrally closed Dubreil-Jacotin inverse semigroup, and partial orderings $\leqq$ on $G$ for which $G$ is a partially ordered group satisfying the condition

(1) $(a, g),(b, h) \in P$ with $a \leqq b$ in $Y, g \leqq h$ in $G$ imply that $a \wedge g c \leqq b \wedge h c$ in $Y$ for each $c \in Y$.

Proof. Suppose that $P(\leqq)$ is an integrally closed Dubreil-Jacotin inverse semigroup and that $\mathscr{R}$ is regular under $\leqq$. From the proof of Theorem 1.6, $\sigma$ coincides with $\mathscr{A}$ on $P$, and so the group $G$ is algebraically isomorphic to the group $P / \mathscr{A}$ under an isomorphism $\psi$, say, which sends $g \in G$ to the $\mathscr{A}$-class $\{(a, g) \in P\} ; \psi$ is well-defined since $g Y \cap Y \neq \square$ for each $g \in G$. For $g, h \in G$, define $g \leqq h$ if and only if $g \psi \leqq h \psi$ in $P / \mathscr{A}$; then $G(\leqq)$ is a partially ordered group.

Let $(a, g),(b, h) \in P$ with $a \leqq b$ in $Y, g \leqq h$ in $G$ and let $c \in Y$. Then by Lemma 1.3,

$$
(b, h)(b, h)^{-1}(a, g)=(b, 1)(a, g)=(b \wedge a, g)=(a, g) \leqq(b, h) .
$$

Further, $(c, 1) \in P$ implies that

$$
(a, g)(c, 1)=(a \wedge g c, g) \leqq(b, h)(c, 1)=(b \wedge h c, h)
$$

and since $\mathscr{R}$ is regular, it follows from [5, Proposition 1.2 (iii)] above that $a \wedge g c \leqq b \wedge h c$ in $Y$, and so $(1)$ is satisfied.

Conversely, suppose that $G(\leqq)$ is a partially ordered group and that (1) holds. On $P$, define $\leqq$ by

$$
(a, g) \leqq(b, h) \Leftrightarrow a \leqq b \text { in } Y \text { and } g \leqq h \text { in } G \text {. }
$$

Then $\leqq$ is a partial ordering on $P$. If $(c, k) \in P$ then

$$
(c, k)(a, g)=(c \wedge k a, k g) \leqq(c \wedge k b, k h)=(c, k)(b, h)
$$

since $k g \leqq k h$ in the partially ordered group $G$, while $k a \leqq k b$ implies that $c \wedge k a \leqq c \wedge k b$ in $Y$. Therefore $\leqq$ is compatible with multiplication on the left in $P$. Also,

$$
(a, g)(c, k)=(a \wedge g c, g k) \leqq(b \wedge h c, h k)=(b, h)(c, k)
$$

since again $g k \leqq h k$ in $G$, and (1) holds. Therefore $\leqq$ is compatible with multiplication on the right, and $P\left({ }^{\prime} \leqq\right)$ is a partially ordered semigroup.

For $(a, g),(c, k) \in P$,

$$
(a, g)(c, k) \leqq(a, g) \Leftrightarrow(c, k) \leqq(1,1)
$$

and so $(a, g) \cdot(a, g)=(1,1)$. Similarly $(a, g) \cdot(a, g)=(1,1)$, and so $P(\leqq \prime)$ is an integrally closed Dubreil-Jacotin inverse semigroup; by definition of $\leqq^{\prime}, \mathscr{R}$ is regular under $\leqq$. 
To complete the proof of the theorem, we have to show that the above correspondence between partial orderings on $P$ and those on $G$ is one-to-one. Suppose therefore that $P(\leqq)$ is an integrally closed Dubreil-Jacotin inverse semigroup, that $\mathscr{R}$ is regular under $\leqq$, and that $(a, g) \leqq(b, h)$ in $P$. Since $\mathscr{R}$ is regular, $a \leqq b$ in $Y$; since the natural map from $P$ onto $P / \mathscr{A}$ is isotone, it follows from the definition of the partial ordering in $G$ that $g \leqq h$. Therefore $(a, g) \leqq{ }^{\prime}(b, h)$. But if $(a, g) \leqq(b, h)$, then $a \leqq b$ in $Y$, and by Lemma $1.3, g \leqq h$ in $G$ implies that

$$
(b, 1)(a, g)=(b \wedge a, g)=(a, g) \leqq(b, h) .
$$

Thus $\leqq$ and $\leqq$ ' coincide on $P$.

On the other hand, given that the partially ordered group $G(\leqq)$ satisfies (1), define $\leqq$ ' on $P$ as above. For $(a, g) \in P, b \in Y$, we have $b \wedge a \leqq b$ and $(b \wedge a, g) \mathscr{A}=(a, g) \mathscr{A}$. Therefore $g \leqq h$ in $G$ if and only if there exist $(a, g) \leqq(b, h) \in P$; by Lemma 1.3, this is equivalent to the existence of $\left(a^{\prime}, g\right) \mathscr{A} \leqq\left(b^{\prime}, h\right) \mathscr{A}$ in $P / \mathscr{A}$, and so the partial ordering induced on $G$ by $\leqq$ coincides with $\leqq$ on $G$. This completes the proof.

There is a corresponding theorem for the case when $\mathscr{L}$ is regular under $\leqq$. The relevant condition to be satisfied is:

$\left(1^{\prime}\right)(a, g),(b, h) \in P$ with $g^{-1} a \leqq h^{-1} b$ in $Y, g \leqq h$ in $G$ imply that $g^{-1}(a \wedge c) \leqq$ $h^{-1}(b \wedge c)$ for each $c \in Y$.

The partial ordering on $P$ then may be defined by:

$$
(a, g) \leqq(b, h) \Leftrightarrow g^{-1} a \leqq h^{-1} b \text { in } Y \text { and } g \leqq h \text { in } G \text {. }
$$

The monotonicity conditions $(1)$ and $\left(1^{\prime}\right)$ are in a sense dual to each other, and in certain circumstances their combination forces $G$ to be trivially ordered; see Proposition 3.4. Yet if $P$ is not $F$-inverse, even (1) and ( $\left.1^{\prime}\right)$ together do not force either $g y \leqq h y$ or $g^{-1} y \leqq h^{-1} y$ when $g \leqq h$ in $G, y \in Y$, as the following example shows.

EXAMPLe 3. Let $Y$ be the closed unit interval of real numbers, with $a \wedge b=\min \{a, b\}$ for $a, b \in Y$. Let $\left\{x_{n}: n \in \mathbb{Z}\right\}$ be distinct elements with $x_{0}=1 \in Y$ and $x_{n} \notin Y$ for $n \in \mathbb{Z} \mid\{0\}$, and let

$$
X=Y \cup\left\{x_{n}: n \in \mathbb{Z}\right\}
$$

Partially order $X$ by

$$
\begin{aligned}
& a \leqq b \text { if } a, b \in Y \text { and } a=a \wedge b, \\
& a \leqq x_{n} \text { for all } a \in Y \mid\{1\}, n \in \mathbb{Z}, \\
& x_{n} \text { is not comparable to } x_{m} \text { for } m \neq n .
\end{aligned}
$$

Then $X$ is a partially ordered set having $Y$ as a subsemilattice ideal, and $Y$ has maximum element 1. Define an action of $\mathbb{Z}$ on $X$ by

$$
\begin{aligned}
n a & =a \text { for } a \in Y \mid\{1\}, n \in \mathbb{Z}, \\
n x_{m} & =x_{m-n} \text { for } m, n \in \mathbb{Z} .
\end{aligned}
$$


Then $\mathbb{Z}$ acts on $X$ by order automorphisms, $X=\mathbb{Z} . Y, n Y \cap Y \neq \square \forall n \in \mathbb{Z}$, and so $P=P(\mathbb{Z}, X, Y)$ is a proper inverse semigroup with identity element $(1,0)$. In fact,

$$
P=\{(a, n): 0 \leqq a<1, n \in \mathbb{Z}\} \cup\{(1,0)\}
$$

and multiplication coincides with multiplication by co-ordinates. The only $\sigma$-class which contains a maximum element is that of $(1,0)$. If we take the usual ordering on $\mathbb{Z}$ and define $\leqq$ on $P$ by co-ordinates also, then ( 1$)$ is satisfied; so is ( $\left.1^{\prime}\right)$, since $P$ is commutative. But $\mathbb{Z}$ does not act trivially on $Y$, and it is not true that $m \leqq n$ in $\mathbb{Z}$ implies that $m 1 \leqq n 1$ or $n 1 \leqq m 1$ in $X$.

3. Strong integrally closed Dubreil-Jacotin inverse semigroups. The conditions (1) and (1') of Section 1 are considerably simplified when $P$ is an $F$-inverse semigroup. By Proposition 1.2 of [5], this is the case when $1 \wedge g 1$ exists for each $g \in G$, and in fact it is equivalent to $X$ being a semilattice [5, Theorem 2.8]. An equivalent condition is given by Proposition 3.1.

Proposition 3.1. Let $P=P(G, X, Y)$ be a proper inverse semigroup with identity element. Then $P$ is an $F$-inverse semigroup if and only if, under its natural partial ordering, $(1,1):(a, g)$ exists for each $(a, g) \in P$.

Proof. For $(a, g) \in P$, there exists a maximum element $(b, h) \in P$ such that

$$
(a, g)(b, h) \preccurlyeq(1,1)
$$

if and only if $h=g^{-1}$ and there exists a maximum element $b \in Y$ such that $b \leqq 1, h^{-1} b=$ $g b \leqq 1$; that is if and only if $1 \wedge g 1$ exists. Since $(1,1)$ is equiquasiresidual, the result follows.

In any inverse semigroup two elements maximum in their respective $\sigma$-classes are comparable under $\preccurlyeq$ if and only if they are equal; this is not necessarily true for an additional partial ordering. Let $P(\leqq)$ be an integrally closed Dubreil-Jacotin semigroup which is also an $F$-inverse semigroup. We shall be interested in the relationships under $\leqq$ between the elements of $P$ maximum in their $\sigma$-classes, and the exact result we shall use is contained in Proposition 3.2. From now on, residuals will be calculated with respect to $\leqq$.

Proposition 3.2. Let $P=P(G, X, Y)$ be an integrally closed Dubreil-Jacotin inverse semigroup. Then $P$ is strong if and only if $P$ is an $F$-inverse semigroup in which

$$
(a, g) \leqq(b, h) \Rightarrow(1 \wedge g 1, g) \leqq(1 \wedge h 1, h) .
$$

Proof. If $P$ is strong then each $\mathscr{A}$-class contains a maximum element. It follows from Theorem 1.6 and its corollary that $P$ is an $F$-inverse semigroup. By definition of $\mathscr{A}$, the above condition is satisfied. then

Conversely, let $(a, g) \in P$. Then $(a, g)(1 \wedge g 1, g)^{-1} \leqq(1,1)$ and if $(a, g)(b, h) \leqq(1,1)$

$$
(a, g)^{-1}(a, g)(b, h) \leqq(a, g)^{-1} \leqq\left(1 \wedge g^{-1} 1, g^{-1}\right)
$$


Since $(a, g)^{-1}(a, g)(b, h) \equiv(b, h)(\mathscr{A})$, it follows that

$$
(b, h) \leqq(1 \wedge h 1, h) \leqq\left(1 \wedge g^{-1} 1, g^{-1}\right)
$$

and that $(1,1):(a, g)=\left(1 \wedge g^{-1} 1, g^{-1}\right)$.

Proposition 3.2 implies that in trying to find which proper inverse semigroups are strong integrally closed Dubreil-Jacotin inverse semigroups, we may concentrate on $F$-inverse semigroups.

Theorem 3.3. Let $P=P(G, X, Y)$ be an F-inverse semigroup. There is a one-to-one correspondence between partial orderings on $P$ under which $\mathscr{R}$ is regular and $P$ is a strong integrally closed Dubreil-Jacotin inverse semigroup, and partial orderings $\leqq$ on $G$ for which $G$ is a partially ordered group satisfying the condition

(2) $g \leqq h$ in $G, c \in Y$ imply that $g c \leqq h c$ in $X$.

Proof. If $\boldsymbol{P}$ is a strong integrally closed Dubreil-Jacotin inverse semigroup in which $\mathscr{R}$ is regular, then there exists a partial ordering $\leqq$ on $G$ for which $G$ is a partially ordered group and (1) of Theorem 2.1 holds. Further, $P$ is $F$-inverse by Proposition 3.2, and from the definition of the partial ordering in $P / \mathscr{A}$, we have $g \leqq h$ in $G$, or equivalently $1 \leqq g^{-1} h$, if and only if

$$
(1,1) \leqq\left(1 \wedge g^{-1} h 1, g^{-1} h\right) \text { in } P \text {. }
$$

This implies that $1 \leqq 1 \wedge g^{-1} h 1$ and so $1=1 \wedge g^{-1} h 1$. Then for $c \in Y$,

$$
(c, 1)=(1,1)(c, 1) \leqq\left(1, g^{-1} h\right)(c, 1)=\left(1 \wedge g^{-1} h c, g^{-1} h\right),
$$

and so $c \leqq 1 \wedge g^{-1} h c$ in $Y$. Hence $c \leqq c \wedge 1 \wedge g^{-1} h c=c \wedge g^{-1} h c$, which implies that $g c \leqq g c \wedge h c$ and finally that $g c \leqq h c$.

Conversely, suppose that $G(\leqq)$ is a partially ordered group for which (2) holds. Then (1) of Theorem 2.1 holds, so that $P$ is an integrally closed Dubreil-Jacotin inverse semigroup as in Theorem 2.1. Further,

$$
(a, g) \leqq(b, h) \text { in } P \Rightarrow a \leqq b \text { in } Y \text { and } g \leqq h \text { in } G \text {, }
$$

and by (2), this implies that $g 1 \leqq h 1$ and so that $1 \wedge g 1 \leqq 1 \wedge h 1$. By Proposition 3.2, we conclude that $P$ is strong, and the result follows.

The condition in Proposition 3.2 is equivalent on an $F$-inverse semigroup $S$ to $\sigma$ being strongly upper regular, in the sense that if $x, y, x^{\prime} \in S$ with $x \leqq y, x \equiv x^{\prime}(\sigma)$, then there exists $y^{\prime} \in S$ such that $y \equiv y^{\prime}(\sigma)$ and $x^{\prime} \leqq y^{\prime}[3$, Chapter 1]. The argument used in Theorem 3.3 may be extended to integrally closed Dubreil-Jacotin inverse semigroups if we assume that $\sigma$ is strongly upper regular. The result is that if $1 \leqq h$ in $G$ then the $\sigma$-class corresponding to $h$ contains a maximum element, and $c \leqq h c$ for each $c \in Y$.

There is a result corresponding to that of Theorem 3.3 for the case in which $\mathscr{L}$ is regular under $\leqq$. The relevant condition to be satisfied is:

(2') $g \leqq h$ in $G$ and $c \in Y$ imply that $g^{-1} c \leqq h^{-1} c$ in $X$. 
In fact, since $g \leqq h$ if and only if $h^{-1} \leqq g^{-1}$, this is equivalent to

$$
g \leqq h \text { in } G \text { and } c \in Y \text { imply that } h c \leqq g c \text { in } X .
$$

Combining this with Theorem 3.3 we have Proposition 3.4, which is essentially Blyth's Theorem 8[1].

Proposition 3.4. The only partial orderings on an F-inverse semigroup $P=P(G, X, Y)$ under which both $\mathscr{R}$ and $\mathscr{L}$ are regular and $P$ is a strong integrally closed Dubreil-Jacotin inverse semigroup, are those for which $g \leqq h$ in $G$ and $c \in Y$ imply that $g c=h c$ in $X$.

EXAmPLE 4. Define an action of $\mathbb{Z}$ on $\mathbb{Z}$ by

$$
n \cdot m=m-n \text {. }
$$

With the usual ordering on $\mathbb{Z}$ this is an action by order automorphisms. The set $\mathbb{Z}^{-}=$ $\{m \in \mathbb{Z}: m \leqq 0\}$ is a subsemilattice and ideal of $\mathbb{Z}$ with maximum element 0 , and since $\mathbb{Z}$ is also a semilattice, $P=P\left(\mathbb{Z}, \mathbb{Z}, \mathbb{Z}^{-}\right)$is a $F$-inverse semigroup. With the usual ordering on the maximum group homomorphic image $\mathbb{Z}$ of $P$, condition $\left(2^{\prime}\right)$ is satisfied, but (2) is not. Therefore, under

$$
(r, s) \leqq(m, n) \Leftrightarrow s \leqq n \text { and } r+s \leqq m+n,
$$

$P$ is a strong integrally closed Dubreil-Jacotin inverse semigroup in which $\mathscr{L}$, but not $\mathscr{R}$ is regular.

In fact one may use [5, Proposition 1.2] to verify that $P$ is a fundamental bisimple inverse semigroup with semilattice of idempotents isomorphic to

$$
\omega=0>-1>-2>\ldots
$$

and so is isomorphic to a transitive inverse subsemigroup of $T_{\omega}$ [9]. But $T_{\omega}$ is isomorphic to the bicyclic inverse semigroup $C(p, q)[4, \mathrm{Vol} .1]$, and so $P$ is isomorphic to $C(p, q)$.

The isomorphism is given by

$$
(m, n) \leftrightarrow q^{-m} p^{-(m+n)}
$$

and in this guise

$$
q^{r} p^{s} \leqq q^{u} p^{v} \Leftrightarrow r-s \leqq u-v \text { and } v \leqq s
$$

\section{REFERENCES}

1. T. S. Blyth, Dubreil-Jacotin inverse semigroups (submitted to Proc. Roy. Soc. Edinburgh).

3. T. S. Blyth and M. F. Janowitz, Residuation Theory, International Series of Monographs in Pure and Applied Mathematics, Volume 102. Pergamon Press, 1972.

4. A. H. Clifford and G. B. Preston, The algebraic theory of semigroups, American Math. Soc. Survey 7, Volumes I and II (Providence, R.I., 1961 and 1967). 
5. D. B. McAlister, Groups, semilattices and inverse semigroups II, Trans. Amer. Math. Soc., 196 (1974), 350-370. 679-688.

6. D. B. McAlister and L. O'Carroll, On B-nomal semigroups, J. London Math. Soc (2), 2 (1970),

7. R. McFadden, On the structure of certain A-nomal semigroups, J. London Math. Soc. (2), 6 (1973), 543-552.

8. R. McFadden and L. O'Carroll, F-inverse semigroups, Proc. London Math. Soc. (3), 22 (1971), $652-666$.

9. W. D. Munn, On fundamental inverse semigroups, Quart. J. Math. Oxford (2), 21 (1970), $157-170$.

10. T. Saito, Proper ordered inverse semigroups, Pacific J. Math. 15 (1965), 649-666.

11. T. Saito, Ordered inverse semigroups, Trans. Amer. Math. Soc. 153 (1971), 99-138.

Department of Mathematical Sciences

NORTHERN ILLINOIS UNIVERSITY

DE Kalb, IllinOIS 60115, U.S.A. 